\title{
Self-Trapping of Light Using the Pancharatnam-Berry Phase
}

\author{
Chandroth P. Jisha, ${ }^{1, *}$ Alessandro Alberucci, ${ }^{1, \dagger}$ Jeroen Beeckman, ${ }^{2}$ and Stefan Nolte ${ }^{1,3}$ \\ ${ }^{1}$ Institute of Applied Physics, Abbe Center of Photonics, Friedrich-Schiller-Universität Jena, \\ Albert-Einstein-Straße 15, 07745 Jena, Germany \\ ${ }^{2}$ Ghent University, ELIS Department, Technologiepark-Zwijnaarde 15, 9052 Gent, Belgium \\ ${ }^{3}$ Fraunhofer Institute for Applied Optics and Precision Engineering, \\ Albert-Einstein-Straße 7, 07745 Jena, Germany
}

(Received 14 November 2018; revised manuscript received 28 March 2019; published 13 June 2019; corrected 18 July 2019)

\begin{abstract}
Since its introduction by Berry in 1984, the geometric phase has become of fundamental importance in physics, with applications ranging from solid-state physics to optics. In optics, the Pancharatnam-Berry phase allows the tailoring of optical beams by a local control of their polarization. Here, we discuss light propagation in the presence of an intensity-dependent local modulation of the Pancharatnam-Berry phase. The corresponding self-modulation of the wave front counteracts the natural spreading due to diffraction; i.e., self-focusing takes place. No refractive index variation is associated with the self-focusing: The confinement is uniquely due to a nonlinear spin-orbit interaction. The phenomenon is investigated, both theoretically and experimentally, by considering the reorientational nonlinearity in liquid crystals, where light is able to rotate the local optical axis through an intensity-dependent optical torque. Our discoveries pave the way to the investigation of a new family of nonlinear waves featuring a strong interaction between the spin and the orbital degrees of freedom.
\end{abstract}

DOI: 10.1103/PhysRevX.9.021051

Subject Areas: Nonlinear Dynamics, Optics, Soft Matter

\section{INTRODUCTION}

The geometric phase is of primary importance in modern physics [1-8]. It arises when a classical or quantum system encompasses a variation of its parameters $[9,10]$, such as a magnetic dipole moving in a rotating magnetic field $[11,12]$. Although initially introduced by Berry in the case of cyclic and adiabatic transformation [11], the geometric phase was soon generalized to parameter variations of any kind $[12,13]$. In optics, the geometric phase was independently discovered by Pancharatnam in 1956 [14] while studying light polarization. Indeed, Pancharatnam found that photons acquire a phase during polarization evolution; this phase depends on the area subtended by the path on the Poincare sphere. This phase, dubbed the PancharatnamBerry phase (PBP), is responsible for a new set of optical phenomena, collectively called spin-orbit photonics $[15,16]$. The PBP, experimentally demonstrated in 1988 in a laser interferometer [17], is not the only phenomenon in classical optics related to the geometric phase: The rotation of the beam polarization caused by a three-

\footnotetext{
*cpjisha@gmail.com

alessandro.alberucci@gmail.com
}

Published by the American Physical Society under the terms of the Creative Commons Attribution 4.0 International license. Further distribution of this work must maintain attribution to the author(s) and the published article's title, journal citation, and DOI. dimensional deflection of the wave vector has been demonstrated in a coiled optical fiber, the latter effect being closely related to the geometric phase concept introduced by Berry [18]. The specific type of phase responsible for this effect is currently called the RytovVladimirskii-Berry phase, and it is strictly related to the spin Hall effect of light in inhomogeneous isotropic materials [16,19]. Other optical effects related to the Berry phase include the Gouy phase [20] and the rotation of images [21].

The action of the PBP manifests clearly when a circularly polarized $(\mathrm{CP})$ beam goes through a half-wave plate: At the output, the helicity is inverted, and a phase, equal to twice the rotation angle of the wave plate, is added to the field. This property has been exploited to design and realize a whole new generation of planar and ultracompact spatial light modulators [22-27]. The influence of the Pancharatnam-Berry phase on light propagation in bulk material has recently been investigated [28,29]. It has been demonstrated that a transverse modulation of the geometric phase yields light confinement, despite the absence of any gradient in the refractive index.

On the other hand, the Kerr effect represents one of the cornerstones in nonlinear optics. The Kerr effect consists of a variation in the refractive index $n$ proportional to the beam intensity $I$. In the spatial domain, a positive Kerr effect (i.e., the higher the intensity, the higher the refractive index) induces self-focusing [30]. For $(2+1) \mathrm{D}$ 
propagation geometries, a pure Kerr effect leads to filamentation and beam collapse [31]. The collapse can be arrested by using nonlocal materials (the nonlinear perturbation expands beyond the illuminated region) or saturable nonlinearities [32-36]. In such cases, a selftrapped beam preserving its shape in propagationusually called a spatial soliton or solitary wave-can be generated [35]. Regardless of the specific type of nonlinearity, light self-trapping implies a point-dependent change in the refractive index of the material, that is, the formation of light-written waveguides [31,36-42]. Solitons in second-order nonlinear materials, substantially based upon an inhomogeneous generation of second harmonics, are a notable exception to this general rule [43]. Because of their huge nonlinearity and large degree of nonlocality, in the last few decades light selftrapping has been investigated thoroughly in nematic liquid crystals (NLCs) [36,44-46]. Essentially, liquid crystals are anisotropic fluids, encompassing properties at an intermediate stage between liquids and solids [47]. In the nematic phase, liquid crystals behave like inhomogeneous uniaxial crystals. The optical axis is parallel at each point to a molecular field, called director $\hat{n}$ [48]. Consequently, optical waves perceive a refractive index $n_{\|}$or $n_{\perp}$ depending on whether electric fields are parallel or normal to the optical axis, respectively.

In this article, we show self-focusing and generation of self-trapped beams based upon a transverse gradient of PBP. We show that the light-induced modulation of the photon polarization (i.e., the spin) affects the field phase, thus changing the local wave vector and, in turn, the beam propagation (i.e., the orbital degree of freedom). Stated otherwise, the observed self-trapping originates from a nonlinear spin-orbit interaction [16]. As nonlinear mechanism, we consider reorientational nonlinearity in NLC, the latter consisting of a rotation of the optical axis proportional to the local optical field $[36,48]$. When the optically induced rotation is coplanar with the wave vector, the extraordinarily polarized beam perceives a self-written change in the refractive index, with no exchange of energy with the ordinary one; this effect has been used to observe self-focusing and self-collimation based upon the dynamic phase in nematic liquid crystals [36,44]. Here, by using a circular polarization capable of exciting both the ordinary and the extraordinary component, we induce a rotation on the plane normal to the wave vector, the latter inducing a power exchange between the ordinary and the extraordinary component. We demonstrate how light is capable of modulating the optical axis on its own, both in the transverse plane and along the propagation direction, leading to the formation of longitudinally periodic structures with a period $\Lambda=\lambda / \Delta n$, where we define the wavelength $\lambda$ and the birefringence $\Delta n=n_{\|}-n_{\perp}$. In turn, such structures can guide light in spite of the absence of gradient in the refractive index [28], the latter corresponding to the dynamic phase of the system [11]. Briefly, the strongly coupled light-matter system undergoes a self-adapting process, finally yielding a stable self-trapped propagation over several Rayleigh lengths.

\section{QUALITATIVE PICTURE OF THE SELF-TRAPPING EFFECT}

The basic mechanism is sketched in Fig. 1. We consider a homogeneous distribution of NLC with the director aligned along the $y$ axis [Fig. 1(a)], illuminated by a CP Gaussian beam propagating along the $z$ direction. We define $\theta$ as the angle formed by the director with the $y$ axis. In the linear regime (i.e., low input powers $P$ ), the optical torque acting on the molecules is negligibly small: The beam diffracts inside the sample, with the polarization evolving periodically with period $\Lambda$ as in a standard wave plate. Thus, in $z=\Lambda / 2$, the beam will be $\mathrm{CP}$ but with opposite helicity with respect to $z=0$, whereas it will be linearly polarized along the diagonal and antidiagonal directions (i.e., forming an angle $\theta \pm 45^{\circ}$ with the axis $x)$ in $z=\Lambda / 4$ and $z=3 \Lambda / 4$. When the input power is increased, molecules undergo an inhomogeneous rotation; i.e., the angle $\theta$ now depends on both the transverse and longitudinal coordinates, respectively [Fig. 1(b)]. Specifically, the rotation $\theta$ will be vanishing when light is $\mathrm{CP}$ (i.e., in $z=0$ and $z=\Lambda / 2$ ), whereas it will reach a maximum (minimum) when $z=\Lambda / 4 \quad(z=3 \Lambda / 4)$, the latter corresponding to the longitudinal positions where the polarization is linear along the diagonal (antidiagonal) direction. The amplitude of the longitudinal modulation of the optical axis varies across the beam cross section owing to the inhomogeneous optical torque. Eventually, this corresponds to a transversely dependent phase modulation due to the net accumulation of PBP [28], in our case leading to the formation of a self-written waveguide. As discussed in Ref. [28] for the linear case, the guiding effect is polarization dependent and the quasimodes of these waveguides undergo a periodic change of their polarization along $z$. Figure 1(c) illustrates the corresponding periodic variation in the Stokes parameter $S_{3}=s_{3} I=-\left(\bar{n} / Z_{0}\right) \operatorname{Im}\left(E_{x} E_{y}^{*}\right)$, where $I=$ $\left[\bar{n} /\left(2 Z_{0}\right)\right]\left(\left|E_{x}\right|^{2}+\left|E_{y}\right|^{2}\right)$ (I is also called the Stokes parameter $\left.S_{0}\right)$ is the field intensity and $\bar{n}=\left[\left(n_{\perp}+n_{\|}\right) / 2\right]$ is the average refractive index. To provide a full description of the light polarization, we also introduce the other two normalized Stokes parameters, $s_{1}=\left[\bar{n} /\left(2 Z_{0}\right)\right]\left(\left|E_{x}\right|^{2}-\left|E_{y}\right|^{2}\right) / I$ and $s_{2}=\left(\bar{n} / Z_{0}\right) \operatorname{Re}\left(E_{x} E_{y}^{*}\right) / I$.

\section{THEORETICAL RESULTS}

\section{A. Ruling equations}

Let us now reformulate the previous qualitative picture in a more formal way. Writing Maxwell's equations in the stationary regime (electromagnetic field $\propto e^{-i \omega t}$ ) in terms of CP waves, the latter being defined as $E_{L}=$ $\left(E_{x}-i E_{y}\right) / \sqrt{2}$ (left circular polarization, LCP) and 
(a)

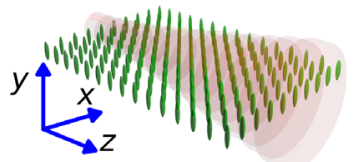

(d)

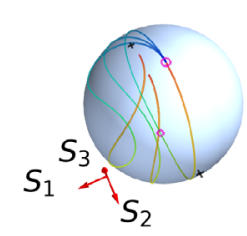

(b)

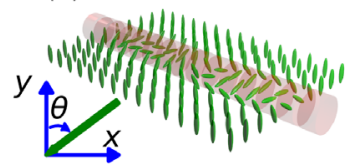

(e)

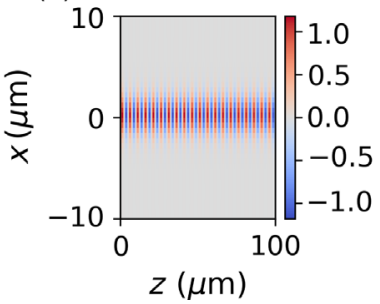

(c)

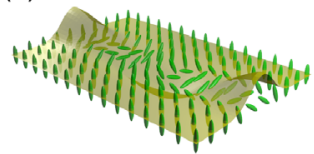

(f)

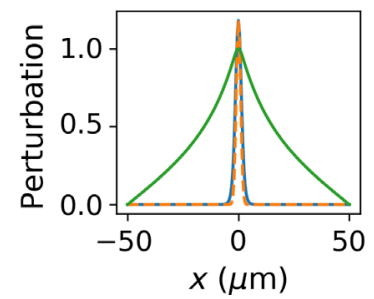

FIG. 1. Schematic of light propagation through a homeotropically aligned LC material. (a) Diffraction of a low-power Gaussian beam propagating through the LC medium. (b) Confinement of a high-power Gaussian beam due to the periodic modulation of the LC molecule. The inset shows the angle $\theta$, which is equal to zero when the optical axis is parallel to the axis $y$. The modulation period is $\lambda / \Delta n$. (c) In each period, the polarization evolves as in a wave plate: The Stokes parameter $S_{3}$ (yellow surface) varies from RCP (input) to LCP $[\lambda /(2 \Delta n)]$ and then back to RCP at the output. (d) Visualization on the Poincaré sphere of the polarization dynamics of plane waves in one birefringent length. The angle of the optical axis $\theta$ is supposed to be sinusoidal along the propagation direction. The different lines correspond to a maximum rotation angle $\theta_{\max }$ of $5^{\circ}, 15^{\circ}$, and $45^{\circ}$, respectively (the loop is almost closed for the lowest angles, but it opens up as the angle is increased). (e) Distribution of the rotation $\theta$ (in degrees) on the plane $x z$ and (f) the corresponding cross section (solid blue line) versus $x$, taken in the section where the rotation angle is maximum; the input parameters are $w=2 \mu$ m, and the power density is $2 \times 10^{3} \mathrm{~W} \mathrm{~m}^{-1}$. The dashed line corresponds to the intensity profile $I$, whereas the green solid curve is the normalized to one light-induced rotation for a uniform (both intensity and polarization) excitation along $z$, i.e., for the case of localization based on the dynamic phase. Here, $\lambda=1064 \mathrm{~nm}, n_{\|}=1.7$, and $n_{\perp}=1.5$.

$E_{R}=\left(E_{x}+i E_{y}\right) / \sqrt{2}$ (right circular polarization, RCP), light propagation for purely transverse fields obeys

$$
\left(\frac{\nabla^{2}}{k_{0}^{2}}+\bar{\epsilon} \mathbb{I}\right)\left(\begin{array}{c}
E_{L} \\
E_{R}
\end{array}\right)+\frac{\epsilon_{a}}{2}\left(\begin{array}{cc}
0 & e^{2 i \theta} \\
e^{-2 i \theta} & 0
\end{array}\right)\left(\begin{array}{c}
E_{L} \\
E_{R}
\end{array}\right)=0
$$

where we defined $\bar{\epsilon}=\left[\left(\epsilon_{\perp}+\epsilon_{\|}\right) / 2\right], \epsilon_{a}=\epsilon_{\|}-\epsilon_{\perp}$ is the optical anisotropy, and $k_{0}$ is the vacuum wave number. Equation (1) shows how the light propagation in the paraxial regime is analogous to a massive particle of unitary magnetic moment interacting with a $\theta$-dependent effective magnetic field $\boldsymbol{B}_{\text {eff }}=\epsilon_{a}[\cos (2 \theta) \hat{x}-\sin (2 \theta) \hat{y}]$ through the coupling energy $\boldsymbol{\sigma} \cdot \boldsymbol{B}_{\text {eff }}$ [49], where $\sigma_{i}$ are the three Pauli matrices. Accordingly, the last term in Eq. (1) accounts for the spin-orbit interaction related to the anisotropic nature of the material. The spin-orbit interaction is spatially varying through the dependence from the rotation angle $\theta$ [1], yielding an inhomogeneous distribution of the geometric phase [11]. If $\theta$ is periodically rotated along $z$ [from numerical simulations, we can factor out the spatial dependences as $\theta(x, y, z)=H(z) \Gamma(x, y)$, with $H(z+\Lambda)=H(z)]$, the PBP accumulates in propagation, and an effective photonic potential $V(x, y)=$ $-\left[\left(2 \pi m\left|\eta_{1}\right|\right) / \Lambda\right] \Gamma(x, y)$ emerges, where $\left|\eta_{1}\right|$ is the amplitude of the fundamental harmonic of $\theta$ and $m$ is the beam helicity with respect to the propagation direction [28].

For plane waves and in the absence of back-reflection, the solution of Eq. (1) can be found by using the
Jones's formalism. Let us make the ansatz $\theta(x, y, z)=$ $\Gamma(x, y) \sin [(2 \pi z) / \Lambda]$, in agreement with the periodic variation of polarization in an anisotropic medium. Figure 1(d) shows, on the Poincaré sphere, the polarization evolution from $z=0$ to $z=\Lambda$ for RCP at the input. For small amplitudes $\Gamma$, the polarization path is very similar to the meridian $S_{1}=0$. When the reorientation increases (i.e., larger input power $P$ ), the path undergoes strong modifications, and the Stokes parameter $S_{1}$ differs significantly from zero.

Equation (1) must be solved in conjunction with the reorientational equation, the latter stating the balancing between the elastic (stemming from intermolecular interactions) and the optical torque [48]. In the static $\left(\partial_{t} \theta=0\right)$ regime, we get

$$
\begin{aligned}
& \frac{1}{\gamma} \nabla^{2} \theta+\frac{2 Z_{0}}{\bar{n}} I(x, y, z)\left[\sin (2 \theta) s_{1}(x, y, z)\right. \\
& \left.\quad+\cos (2 \theta) s_{2}(x, y, z)\right]=0
\end{aligned}
$$

where we have defined the light-matter coupling constant $\gamma=\left[\left(\epsilon_{0} \epsilon_{a}\right) /(4 K)\right]$, with $K$ being Frank's elastic constant [48]. According to Eq. (2), the nonlinear response of the system is nonlocal; that is, $\theta$ changes even in regions where the intensity vanishes $[33,36,50]$. Differently from most of the nonlocal nonlinear media, Eq. (2) shows that in LCs the relationship between the field intensity $I$ and the nonlinear director rotation $\theta$ is nonlinear. As a direct consequence, the 


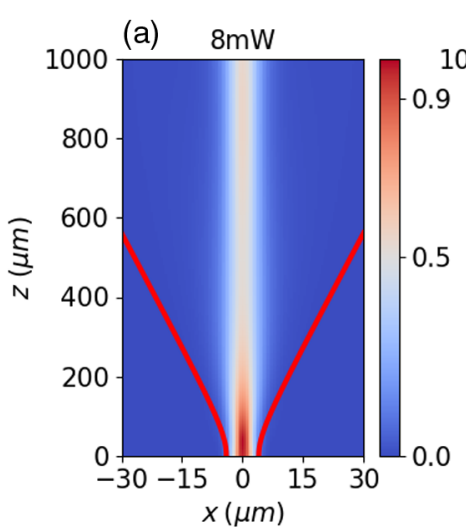

(b) $s_{1}$
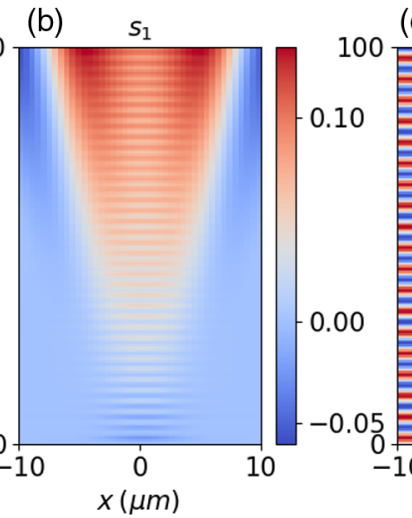

(c)

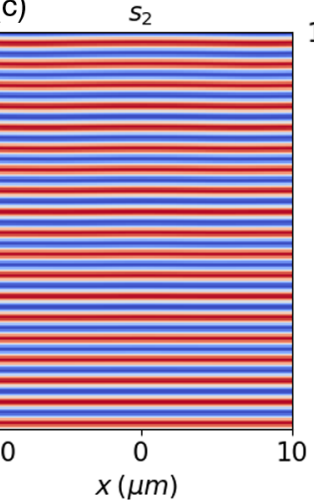

(d)
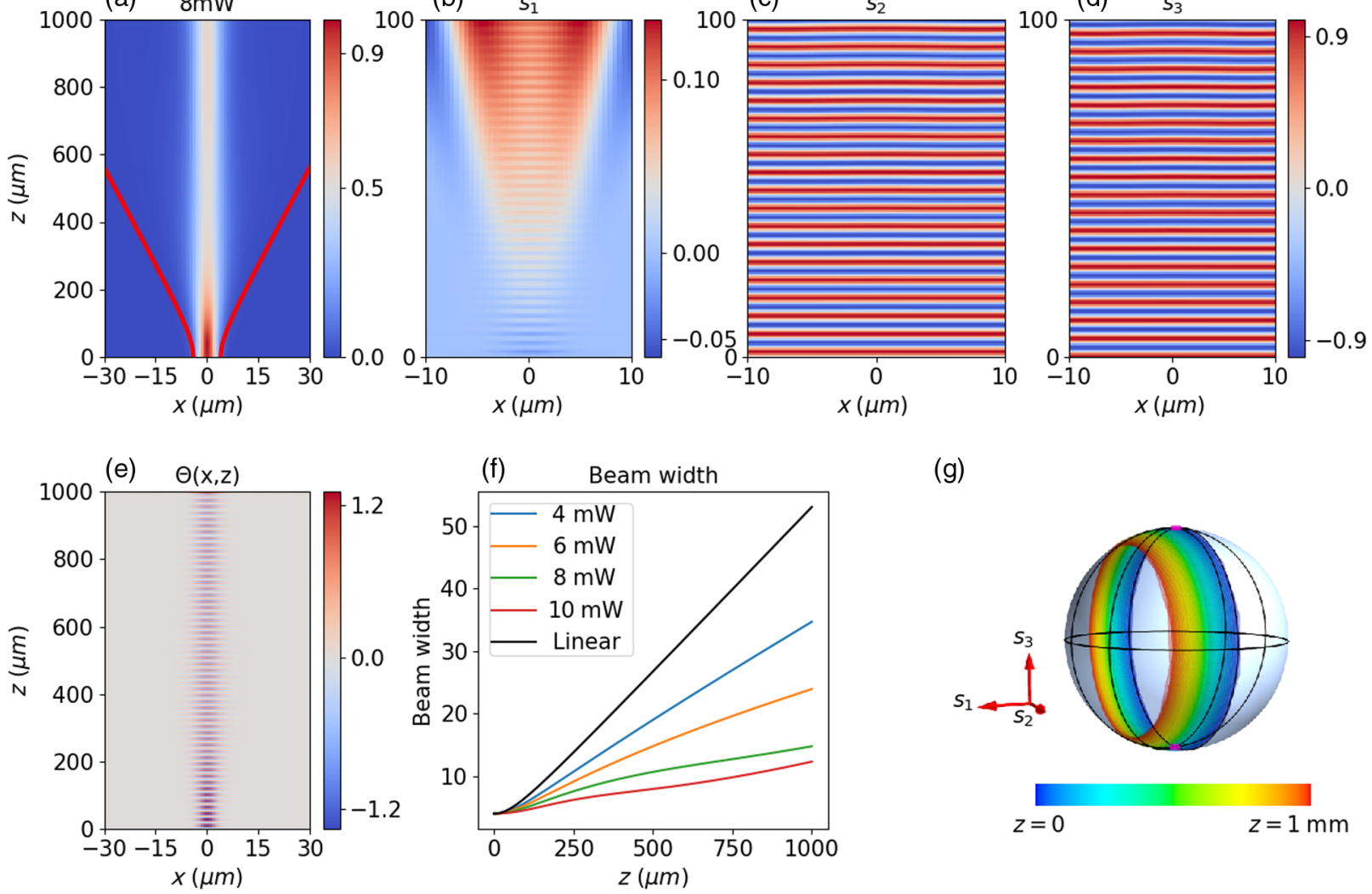

(g)

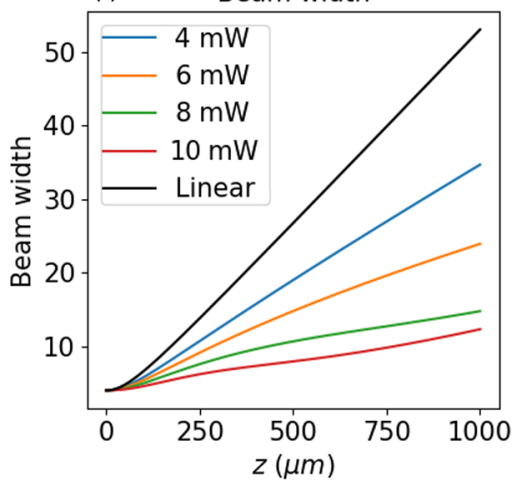

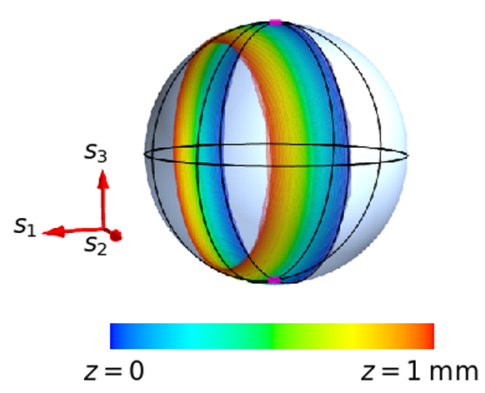

FIG. 2. Numerical simulations for a Gaussian beam of input width $w_{\text {in }}=4 \mu \mathrm{m}$. (a) Evolution of a high-power $(\mathcal{P}=8 \mathrm{~mW}) \mathrm{Gaussian}$ beam propagating through a homeotropically aligned NLC cell. The red solid lines show the linear diffraction. (b)-(d) Polarization evolution of the beam, both in the transverse and longitudinal direction, shown in terms of the normalized Stokes parameters $s_{1}, s_{2}$, and $s_{3}$. (e) Corresponding light-induced periodic rotation of the optical axis resulting in the confinement of the beam. (f) Evolution of the width of the beam with decreasing power (bottom to top curve). The topmost curve shows the linear diffraction. (g) Mapping on the Poincaré sphere of the dynamics along $z$ of the polarization state measured at the center of the Gaussian beam. The blue (red) color corresponds to small (large) propagation distance $z$ (check the color bar below the Poincaré sphere for the chromatic gauge). Here, the input polarization is RCP, $\lambda=1064 \mathrm{~nm}, n_{\|}=1.7$, and $n_{\perp}=1.5$.

solution of Eq. (2) strongly depends on the input polarization through the Stokes parameters [51]. For example, if the light polarization is normal to the director at rest, linearly polarized inputs move the director only when the impinging power overcomes the Fréedericksz threshold [48]. A typical solution of Eq. (2) in $(1+1) \mathrm{D}$ is shown in Fig. 1(e), where we suppose a diffraction-less beam with polarization varying as $s_{3}=\cos \{[(2 \pi) / \Lambda] z\}$ and $s_{2}=\sin \{[(2 \pi) / \Lambda] z\}$. As anticipated in our preliminary discussion, the rotation angle $\theta$ follows $s_{2}$; that is, molecules are periodically rotated by the optical field. Figure 1(f) compares the cross section of $\theta$ (blue solid lines) with respect to the optical intensity $I$ (orange dashed lines). The degree of nonlocality is very small with respect to standard reorientational solitons in NLCs, where the nonlocality is fixed by the size of the sample (green solid line) $[36,39]$. The difference is easily explained by noticing that, for small $\theta$, Eq. (2) is analogous to a Poisson's equation ruling electrostatics [39]. As a matter of fact, the periodic forcing term (proportional to $S_{2}$ ) corresponds to a vanishing overall charge; thus, the long-range field along the transverse direction must vanish.

\section{B. Numerical simulations}

We run numerical simulations in $(1+1) \mathrm{D}$ of the system composed by Eqs. (1) and (2) to verify our basic idea (see the Appendix B for the technical details). The intensity and the corresponding Stokes parameter distribution are plotted in Figs. 2(a)-2(d). As predicted, for large enough powers (in the plotted case, the effective power is $\mathcal{P}=8 \mathrm{~mW}$; see Appendix $\mathrm{B}$ for the definition of $\mathcal{P}$ ), the optical beam rotates the molecules in a periodic fashion [Fig. 2(e)], inducing a strong optical self-focusing due to the accumulation of the geometric phase in propagation. The extension across $x$ of the optical perturbation is of a few microns, in accordance with Figs. 1(e) and 1(f). The larger the input power, the stronger the self-focusing, as shown by the trend of the beam width $w$ versus the propagation distance $z$ and the optical power $\mathcal{P}$ plotted in Fig. 2(f). 

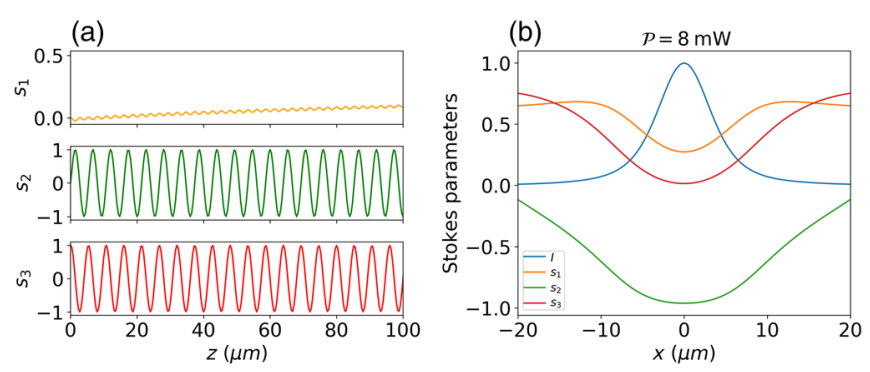

FIG. 3. Polarization structuring of Pancharatnam-Berry solitons. (a) Stokes parameters $s_{1}, s_{2}$, and $s_{3}$ versus the propagation distance $z$ in the beam center $x=0$. The evolution in the first $100 \mu \mathrm{m}$ in a 1-mm-long cell is plotted. (b) The corresponding transverse distribution of the four Stokes parameters versus $x$ computed in $z=0.5 \mathrm{~mm}$. Here, $\mathcal{P}=8 \mathrm{~mW}$.

Figure 2(g) maps, on the Poincaré sphere, the polarization in $x=0$ as it evolves along $z$, including the whole interval from $z=0$ to $z=1 \mathrm{~mm}$, with each color corresponding to a different propagation distance $z$. In agreement with the plane-wave model plotted in Fig. 1(d), the Stokes parameter $s_{1}$ is negligible due to the smallness of $\theta$. But as the light propagates inside the nonlinear medium, a more complicated polarization state emerges, the latter showing features beyond the plane-wave model: Despite the smallness of the rotation angle $\theta$, the motion on the Poincare sphere corresponds to a constant increase in $s_{1}$, whereas the other two Stokes parameters $s_{2}$ and $s_{3}$ trace periodic orbits on the sphere. The dynamics versus $z$ for $z<100 \mu \mathrm{m}$ of the Stokes parameters sampled in the beam center is plotted in Fig. 3(a). Figure 3(b) shows the transverse distribution (i.e., in the plane normal to $z$ ) of the three Stokes parameters. Because of the complicated interaction between light and matter, the polarization of the nonlinear waves changes significantly along the beam cross section: This change demonstrates that the formation of a so-called structured beam [52] is required for self-trapping to occur.

The emergence of structured beams (i.e., polarization varying along both $x$ and $z$ ) in the nonlinear regime is predicted by the plane-wave model as well: In fact, in anisotropic materials that are transversely homogeneous but subject to periodic rotation along the longitudinal direction, a nonvanishing $s_{1}$ features the plane-wave quasieigenstates of the system [see Fig. 1(d)]. Physically speaking, there is a nonlinear exchange of angular momentum between matter and light [51], the latter counteracting the natural diffraction-induced spreading of light owing to the strong spin-orbit coupling of the system [16,23].

\section{EXPERIMENTS}

\section{A. Single beam at normal incidence}

In order to verify our theoretical predictions, we coupled a circularly polarized fundamental Gaussian beam at $\lambda=$ $1064 \mathrm{~nm}$ inside a NLC planar cell of thickness $75 \mu \mathrm{m}$ along $y$, and much larger sizes along $x$ and $z$. To achieve the vertical alignment shown in Fig. 1(a), we took a cell with the director initially aligned along the $z$ direction and then applied a sinusoidal voltage of amplitude $9 \mathrm{~V}$ (see Appendix C). This choice was made to minimize the presence of (technologically unavoidable) defects in the molecular alignment, which could inhibit the observation of the PBP-induced light trapping due to the smallness of the required rotation angle, the latter being on the order of a few degrees [see Fig. 2(e)]. In fact, owing to the saturation in the reorientational equation, potential inhomogeneities present in the rubbing distribution (corresponding to a point-dependent boundary condition for the angle $\theta$ when solving the reorientational equation) are washed out by the application of a large enough voltage [53].

The intensity distribution on the plane $x z$ can be directly visualized by collecting the scattered light with a customized microscope, the minimum available resolution in our case being fixed by the diffusion of photons in liquid crystals. Results are plotted in Figs. 4(a) and 4(b). For low power, the beam diffracts linearly. As the power is increased $(P=10 \mathrm{~mW})$, self-focusing is observed, the latter manifesting as a weaker light spreading. At higher powers, diffraction is compensated, and a self-collimated beam is excited inside the sample. The behavior of the beam width versus $z$ [Fig. 4(c)] is in agreement with the simulations reported in Fig. 2(f). Discrepancies at short propagation distances are ascribed to diffusive photons emitted in correspondence to the input interface. The intensity cross sections encompass exponential tails, the latter increasing in magnitude with $z$ [Fig. 4(d)]. Such exponential tails are related to the intrinsic timedependent disorder of NLCs (see Appendix E). The light self-trapping is evident by comparing the observed propagation with respect to the linear case. The reorientational origin of the phenomena is also proved by the dependence of self-focusing on the direction angle of the linear input polarization: In fact, the maximum self-focusing effect is achieved for input polarizations around the diagonal directions [53].

Generally speaking, a rotation of the director can be associated with both the dynamic [36] and the geometric phase. In the ideal case of a wave vector (taken parallel to $\hat{z}$ ) normal to the director and the latter perfectly aligned along $y$ inside the sample, light is subject solely to gradients in the geometric phase, given that the director can rotate only on the plane $x y$ [28]. Let us discuss the accuracy of this assumption in our experiments. Two main issues can invalidate our hypothesis: misalignment of the director and tilt of the wave vector with respect to the ideal $z$ direction. With respect to the former, the applied bias induces the director to form an angle of $89.9^{\circ}$ with respect to the plane $x z$, corresponding to a maximum refractive index jump of approximately $6 \times 10^{-7}$ for rotation in the $y z$ plane, as in standard solitons in a biased cell [36]. This 

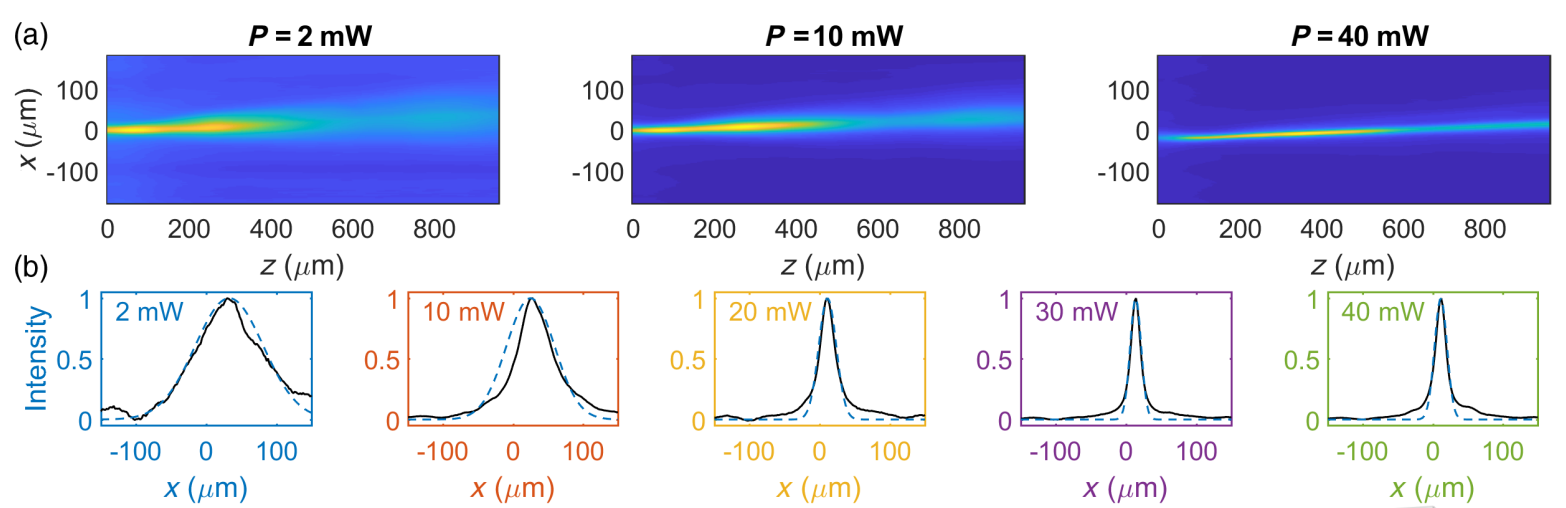

(c)

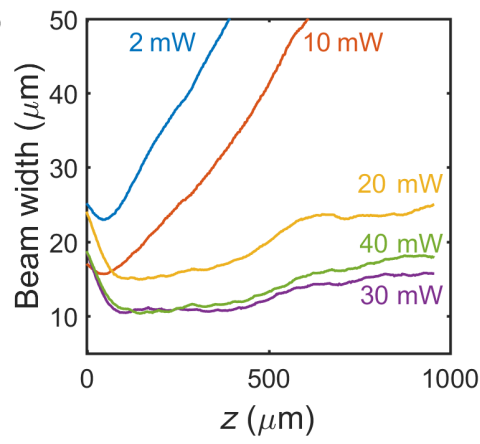

(d)

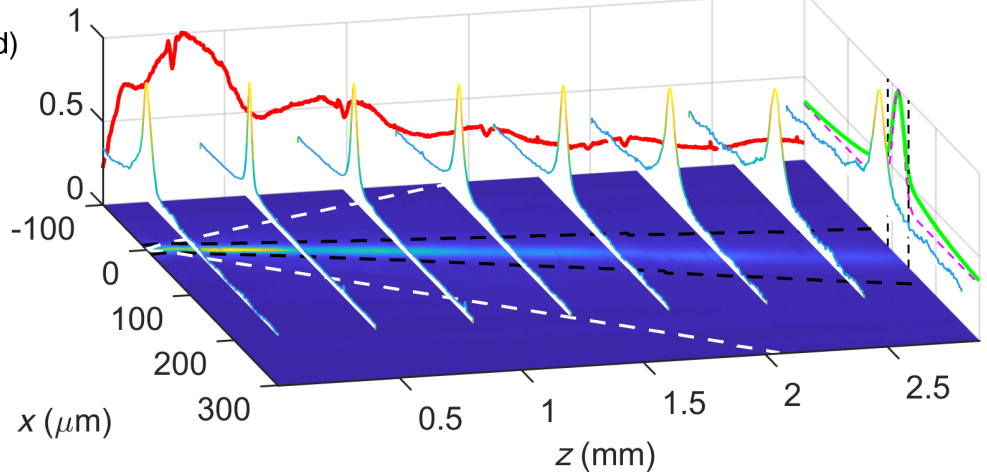

FIG. 4. Experimental evidence of light self-localization. (a) Light distribution on the plane $x z$ for $P=2 \mathrm{~mW}$ (linear diffraction), $10 \mathrm{~mW}$ (mild self-focusing), and $40 \mathrm{~mW}$ (self-trapping). (b) Collection of the beam cross sections at $z=900 \mu \mathrm{m}$ for several input powers. The black solid and blue dashed lines are the acquired profile and the corresponding best-fitting Gaussian function, respectively. (c) Beam width versus $z$ for the set of powers shown in (b). (d) Light evolution when $P=30 \mathrm{~mW}$, the latter corresponding to the best confinement [see panel (c)]. The propagation is shown for over $2.5 \mathrm{~mm}$ by collaging three different pictures. The dashed lines are the beam width for Gaussian beams with waists of $2 \mu \mathrm{m}$ (white lines) and $10 \mu \mathrm{m}$ (black lines). The red solid line is the behavior of the intensity peak versus $z$. The green solid (magenta dashed) line is the best fit to the final beam cross section, accounting for (disregarding) the photon diffusion. The wavelength is $\lambda=1064 \mathrm{~nm}$, and the input waist is $2 \mu \mathrm{m}$, placed in $z=0$.

refractive index gradient corresponds to a nonlinear mode approximately $3 \mathrm{~mm}$ wide, thus not significantly affecting the observed self-confinement (see Appendix C for further details). Let us now discuss possible tilts in the input wave vector. Significant tilts with respect to the $y z$ plane can be excluded: We are able to see the light throughout the sample of length $2 \mathrm{~cm}$ (self-trapping after a given distance is lost due to the scattering losses), the latter implying a vertical angular accuracy of about $0.1^{\circ}$ given the thickness of the cell. With respect to the $x z$ plane, an accuracy of about $0.2^{\circ}$ was achieved by overlapping the trajectories of the extraordinary and of the ordinary beam in the linear regime over a length of about $1 \mathrm{~mm}$. Summarizing, for the angle between the wave vector and the director, the wavefront tilt induces an error with respect to the ideal case of $90^{\circ}$ comparable to our accuracy in the alignment of the optical axis $\hat{n}$ itself. Accordingly, the magnitude order of the overall error in the measured nonlinear changes in the refractive index is $10^{-7}$; hence, it does not significantly affect the observed self-trapping.

On the other hand, we can also rule out any significant contribution from the thermo-optical effect to the selftrapping. For horizontal (polarization along $x$, corresponding to the ordinary wave) and for vertical (polarization parallel to $\hat{y}$, thus exciting the extraordinary component into the NLC bulk) linear polarizations, in our configuration the thermal nonlinearity induces a focusing and a defocusing effect, respectively [54], with the defocusing effect being about 2 times stronger than the focusing one. In the presence of a circularly polarized input, we thus expect a net defocusing effect, which means that the thermo-optical effect is hindering the self-trapping. Experimentally speaking, in the Supplemental Material, we show that no appreciable defocusing effect is observed for input linear beams polarized along $y$ [53].

\section{B. Single beam at oblique incidence}

To confirm the geometric phase origin of the observed self-confinement, we experimentally investigated light propagation when the input wave vector is tilted by $4^{\circ}$ on the $x z$ plane with respect to the normal at the input interface. Because of the birefringence of the NLC, the ordinary and the extraordinary beams propagate along different trajectories in the linear regime, i.e., at low power. Figure 5 shows the observed intensity distribution inside 


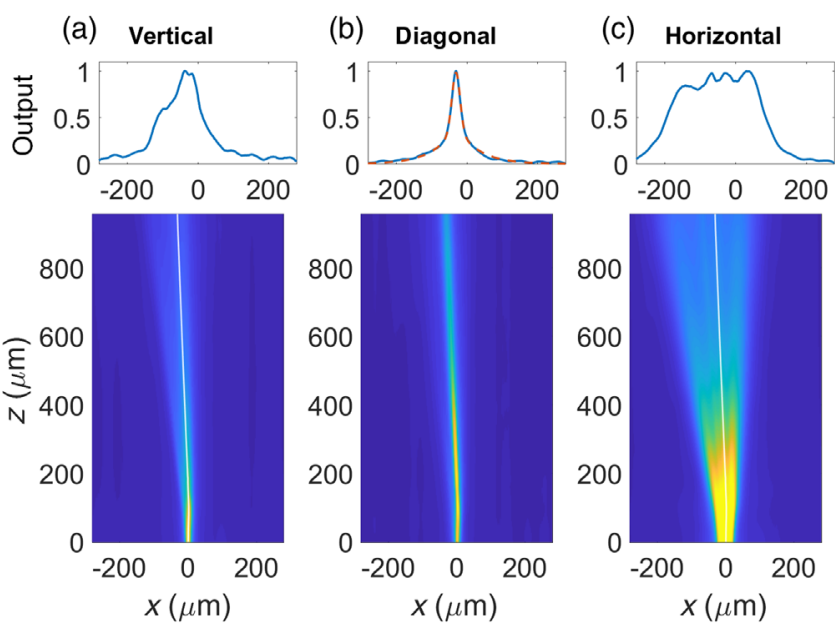

FIG. 5. Light behavior for tilted input. Light propagation when the input beam is tilted by approximately $4^{\circ}$ with respect to the normal at the input interface and is linearly polarized (a) parallel to axis $y,(b)$ at $45^{\circ}$, and (c) parallel to the axis $x$. The top panels report the intensity cross section at $z=960 \mu \mathrm{m}$ (solid blue line), whereas the dashed red line is the best fit accounting for light diffusion (see Appendix E), providing a width of $20 \mu \mathrm{m}$ to be compared with the value of approximately $50 \mu \mathrm{m}$ measured in the linear case. The solid white line in (a) and (c) is the trajectory corresponding to the self-trapped beam plotted in (b). The input power is $50 \mathrm{~mW}$, and the input waist is $4 \mu \mathrm{m}$.

the sample for three different linear polarizations when the input power is fixed at $50 \mathrm{~mW}$. When the input is vertical [polarization along $y$, plotted in Fig. 5(a)], no appreciable nonlinear effects are observed, given that only an extraordinary component parallel to the director $\hat{n}$ is excited in the NLC, resulting in a vanishing optical torque applied to the molecules. When the polarization is horizontal [polarization along $x$, plotted in Fig. 5(c)], only the ordinary component is propagating inside the sample in the linear regime. When the input power is increased, because of the oblique incidence, the horizontal input causes a light-driven local mixing of ordinary and extraordinary components at the input interface, in turn resulting in a strong defocusing effect generated at the input interface [53] itself. Hence, in this configuration, the thermal focusing is negligible, analogously to the normal incidence case discussed in Sec. IVA [54]. Conversely, for diagonal polarizations [Fig. 5(b)], the joint action of the two components yields a substantial molecular rotation in the $x y$ plane, in turn generating a PBP gradient capable of spatially locking the two components and generating a vector self-trapped beam via the spin-orbit coupling. On the one hand, the dependence on the input polarization and the necessity for the simultaneous presence of both the linear components at the input confirms the reorientational origin of the selftrapping, governed by Eq. (2) and thus strongly nonlinear with respect to all four Stokes parameters of the input beam. On the other hand, the available variation in the dynamic phase is much bigger (approximately 4 orders of magnitude) than for normal incidence: Nevertheless, no appreciable self-focusing is observed for vertical inputs, the latter corresponding to the maximum light-induced refractive index gradient. At the same time, for diagonal inputs, we observe that all the light (including the ordinary component) is self-trapped, a phenomenon that cannot be explained by refractive index gradients given that the ordinary refractive index $n_{\perp}$ is invariant with respect to any rotation of the optical axis. Finally, a lower confinement is observed for circular polarization due to the depolarizing effect coming from the region adjacent to the input interface [53].

\section{Interaction between two beams}

To provide further proof of the PBP origin of the selfconfinement, we finally investigated the interaction between two beams encompassing an opposite helicity. When the helicity of the input beam is switched, the periodic perturbation of the director is shifted by a halfperiod, resulting in a photonic potential inverted in sign (see Appendix A). The interaction between the two beams is then repulsive, analogously to two electric charges of opposite sign [39]. Numerical results shown in Fig. 6 confirm this behavior: The two beams repel reciprocally: the closer the beams, the stronger the repulsion is. It is well known that, in the presence of a coherent nonlinear mechanism, the interaction between spatial solitons can be changed from attractive to repulsive by changing the relative phase [35]. Nonetheless, reorientational nonlinearity in NLC is incoherent (i.e., depends on the beam intensity) and, in the dynamic case, is characterized by a (a)

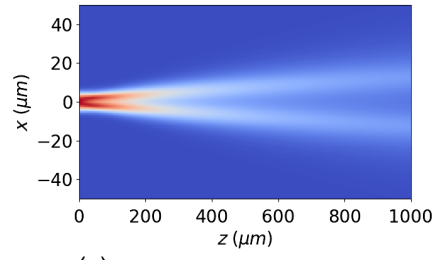

(c)

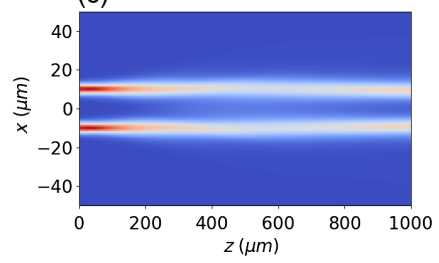

(b)

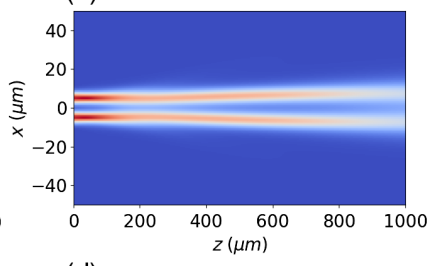

(d)

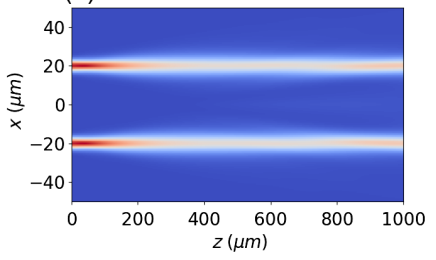

FIG. 6. Simulations of the two-beam interaction. Overall intensity distribution calculated on the plane $x z$ when the initial distance is (a) $4 \mu \mathrm{m}$, (b) $10 \mu \mathrm{m}$, (c) $20 \mu \mathrm{m}$, and (d) $40 \mu \mathrm{m}$. The two beams are both circularly polarized but with inverted spin. The input waist is $4 \mu \mathrm{m}$, the wavelength is $1064 \mathrm{~nm}$, the cell length along $z$ is $1 \mathrm{~mm}$, and the input power is $\mathcal{P}=8 \mathrm{~mW}$ on each beam. 

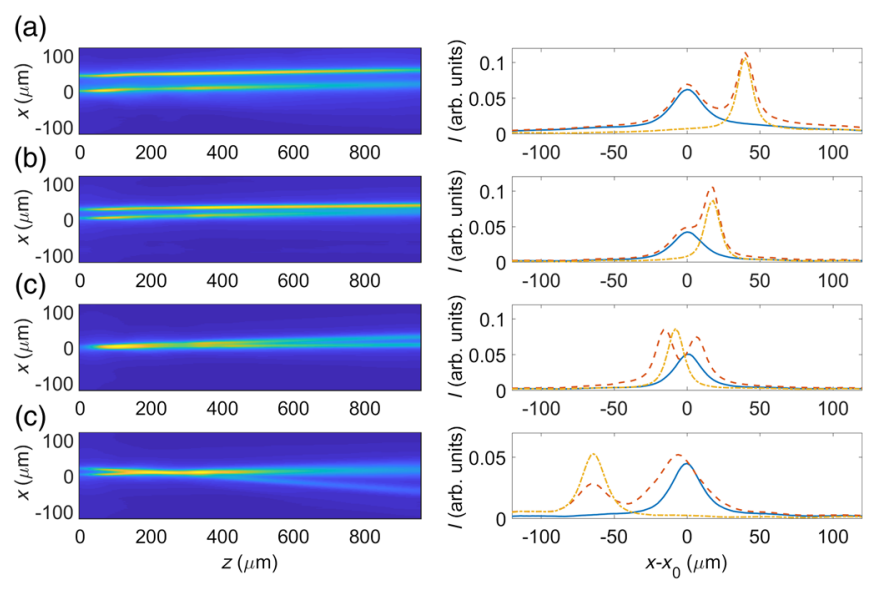

FIG. 7. Two-beam interaction with only the geometric phase. (a)-(c) Interaction of two parallel optical beams separated by a distance of (a) $40 \mu \mathrm{m}$, (b) $20 \mu \mathrm{m}$, and (c) $0 \mu \mathrm{m}$. In (d) the upper beam is tilted in order to achieve an X-junction configuration. The first column illustrates the intensity distribution on the observation plane $x z$, whereas the second column reports the intensity cross section at $z=960 \mu \mathrm{m}$ when the two beams are launched together (orange dashed lines) or when only the upper or the lower beam is launched alone (dot-dashed yellow and blue solid lines, respectively). In the right column, $x_{0}$ is the position of the lower beam when propagating alone. The input waist is $4 \mu \mathrm{m}$, whereas the input powers are $50 \mathrm{~mW}$ for the top beam and $40 \mathrm{~mW}$ for the bottom beam.

large degree of spatial nonlocality [36,55], ruling out this possibility. The absence of dependence on the relative phase was confirmed in our experiments, where we observed that the sign of the attraction was independent from the difference in the optical path between the two beams before entering the cell.

Numerical simulations show another peculiar feature of PBP-based solitons: The interaction length between solitons is short range (less than $20 \mu \mathrm{m}$ ), in agreement with the transverse extension of the optically induced perturbation in the director plotted in Figs. 1(e) and 1(f). This behavior, strikingly different from standard solitons in NLC [36,56], can be intuitively understood by considering the reorientation equation (2). In fact, such an equation for small rotation angle $\theta$ can be recast as

$$
\nabla^{2} \theta+\frac{2 \gamma Z_{0}}{\bar{n}} I(x, y, z) s_{2}(x, y, z)=0,
$$

which is mathematically analogous to the Poisson equation [39,57] providing the electrostatic potential (in our analogy, the angle $\theta$ ) once the distribution of electric charges is known (in our case, the Stokes parameter $S_{2}=I s_{2}$ ). Effectively, we have a distribution of charges localized around the beam axis. Thus, the angle $\theta$ for transverse positions $(x, y)$ away from the beam will be vanishing only if the average value of $S_{2}$ is null; i.e., $S_{2}$ needs to be periodic or quasiperiodic,
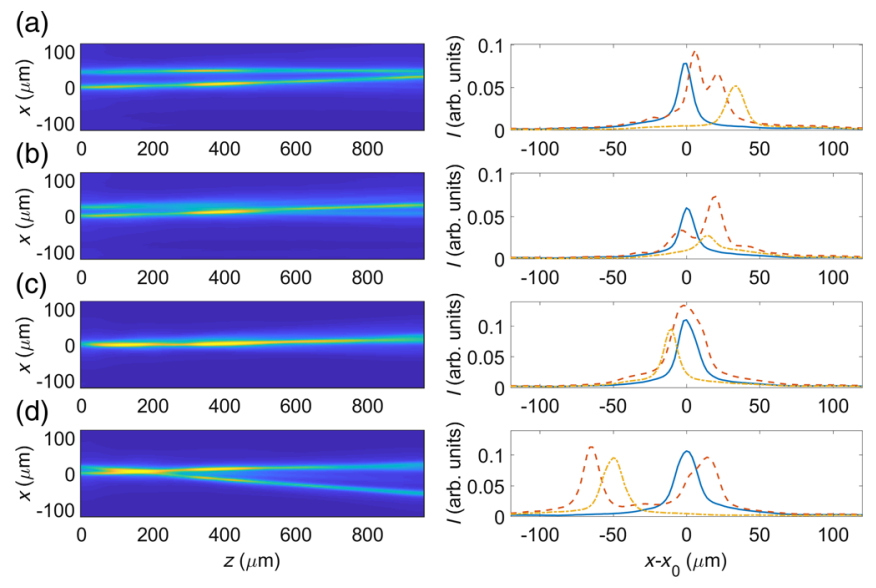

FIG. 8. Two-beam interaction in the presence of both the geometric and the dynamic phase. The input beams are the same as those launched in Fig. 7, but now a lower voltage of $2 \mathrm{~V}$ is applied to the sample [(a)-(c)]. The input powers are now (a) 5 and $4 \mathrm{~mW}$, (b) 4 and $3 \mathrm{~mW}$, (c) 8 and $7 \mathrm{~mW}$, and (d) 7 and $6 \mathrm{~mW}$.

in agreement with Fig. 1(e). To summarize, the short-distance interaction is a direct proof of the $z$-periodic rotation of the optical axis induced by light.

Experimentally, we split the input beam into two halves via a Mach-Zehnder interferometer. The setup is designed to ensure opposite helicities for the two beams impinging on the sample (see Appendix D). The interaction is investigated for different positions and propagation angles of one of the beams; the corresponding results are shown in Fig. 7. When the relative distance is $40 \mu \mathrm{m}$ [Fig. 7(a)], no interaction is observed. When the initial distance is $20 \mu \mathrm{m}$ [Fig. 7(b)], a tiny shift of $2 \mu \mathrm{m}$ (corresponding to the pixel size in our visualization system) is observed on the upper beam (i.e., the beam placed in $x>0$ ). The lack of a significant interaction is in agreement with the weak nonlocality associated with Eq. (3). When the tails of the two fields touch each other at the input [Fig. 7(c)], the distance between the two beams increases with the propagation distance, demonstrating that a net repulsion is at work. Owing to the lack of significant interaction after the overlapping region, the two beams propagate along straight lines conserving the self-confinement, in agreement with Fig. 6(a). Figure 7(d) finally shows the interaction when one of the beams is tilted with respect to the reference beam. After the crossing point, the relative distance between the two beams slightly diminishes due to the interaction. The interaction also affects the self-trapping, with a small widening of the self-trapped waves.

To test our idea, we repeated the two-beam experiment shown in Fig. 7 at the same point and input conditions (only the input powers are changed, see below), but we applied a lower voltage to the cell $[36,55,56]$. The angle between the director and the vertical component of the optical electric field now differs significantly from zero, 
leading to a rotation of the optical axis on the $y z$ plane $[36,45]$, the latter corresponding to a refractive index gradient. In other words, the nonlinear propagation is now determined by the interplay between the dynamic and the geometric phase. Results are reported in Fig. 8. The voltage is chosen low enough to ensure a dominance of the dynamic over the geometric phase. The input power required for self-trapping is much lower than for the PBP case (about 10 times smaller). In agreement with the highly nonlocal response in space [36,55], the beams attract each other, even when the distance is $40 \mu \mathrm{m}$ [see Figs. 8(a) and 8(b)]. When the two beams are launched in the same position, they merge into a single beam [see Fig. 8(c)]. Finally, in the $\mathrm{X}$-junction configuration shown in Fig. 8(d), the distance between the two beams increases; that is, the behavior is opposite with respect to the PBP case shown in Fig. 7(d). This dynamics confirms that the sign of the interaction between self-trapped beams based upon the geometric and the dynamic phase in NLC is the opposite.

\section{CONCLUSIONS AND FUTURE PERSPECTIVES}

In conclusion, we demonstrated the self-focusing and self-confinement of electromagnetic waves via the Pancharatnam-Berry phase. The fundamental mechanism is based upon spin-orbit interaction and thus strictly connected to gauge fields [58]. The nonlinear waves studied here do not carry orbital angular momentum; however, because of the nonlinear spin-orbit interaction, we envisage the existence of a larger family of self-trapped fields encompassing a power-dependent exchange of spin and orbital angular momentum [59]. Future advancements of our work also include the investigation of the connection with waveguiding based upon inhomogeneous gauge fields, theoretically proposed in Ref. [60] and recently demonstrated in a waveguide array [61]. On the experimental side, we envisage the observability of PBP-based optical self-trapping in solutions filled up with anisotropic dopants, prealigned by the application of external symmetrybreaking stimuli [41,62-64]. Another interesting generalization of our work is to add the temporal degree of freedom by using a modulated $\mathrm{CW}$ or pulsed laser [65]. Similar phenomena are also expected to occur in other systems, such as Bose-Einstein condensates or beams of charged particles under the action of a magnetic field, real or effective. With respect to basic physics, our work confirms the relevancy of liquid crystals as a platform for the investigation of angularmomentum exchange between light and matter in the nonlinear regime [66,67], including spin-to-orbital conversion and the generation of self-trapped structured beams. The selflocalization provides a novel platform to create reconfigurable all-optical devices like directional couplers, beam splitters, beam combiners, and so on, based on the Pancharatnam-Berry phase and in the absence of refractive index modulation. On the technological side, we also envisage the application of these results to the generation of permanent Pancharatnam-Berry waveguides after polymerization of the liquid crystal.

\section{ACKNOWLEDGMENTS}

We thank Frederik Van Acker for his help in the laboratory and Dr. Raouf Barboza for critical reading of the manuscript. A. A. is supported by Deutsche Forschungsgemeinschaft (DFG) via the International Research Training Group GRK 2101.

C. P. J. and A. A. contributed equally to this work.

\section{APPENDIX A: LIGHT PROPAGATION IN A TWISTED LIQUID CRYSTAL}

At optical frequencies, NLCs are nonmagnetic inhomogeneous uniaxial crystals, with the optical axis corresponding to the symmetry axis, named the director $\hat{n}(\boldsymbol{r})$. For fixed external excitations and given boundary conditions, the spatial distribution of $\hat{n}$ in the stationary regime is determined by the balance between all the torques acting on the molecules [48]. The equilibrium is achieved when the elastic torque balances out the optical one, where the elastic and the optical torques correspond to the first term $\left(\propto \nabla^{2} \theta\right)$ and the second term $(\propto I)$ in Eq. (2), respectively. Once the director distribution is known, the optical properties are then determined by the director orientation and by the dielectric eigenvalues $\epsilon_{\perp}$ and $\epsilon_{\|}$, the latter being valid for electric fields oscillating normal and parallel to the optical axis, respectively. In fact, the dielectric tensor $\boldsymbol{\epsilon}$ is given by $\epsilon_{i j}=\epsilon_{\perp}+\epsilon_{a} n_{i} n_{j} \delta_{i j}$, where $\epsilon_{a}=\epsilon_{\|}-\epsilon_{\perp}$ is the optical anisotropy. In our theoretical discussion, we consider that the boundary conditions at the cell edges induce a homeotropic alignment of the molecules along the $y$ axis all around the sample [see Fig. 1(a)].

We consider optical beams impinging normally to the cell, thus having a wave vector parallel to $\hat{z}$. In the paraxial limit, the electromagnetic field lies in the $x y$ plane. When illuminated, the NLC molecules can rotate only in the xy plane due to the paraxial nature of the field. Thus, the optical properties are fully determined by the rotation angle $\theta$ with respect to the rotation axis $z$. In the stationary regime (electromagnetic field $\propto e^{-i \omega t}$, wavelength $\lambda$, and wave number $k_{0}=2 \pi / \lambda$ ), evolution along $z$ of the electric field $\boldsymbol{E}=E_{x} \hat{x}+E_{y} \hat{y}$ is ruled by [68]

$$
\left(\frac{\nabla^{2}}{k_{0}^{2}}+\epsilon_{\perp} \mathbb{I}\right)\left(\begin{array}{c}
E_{x} \\
E_{y}
\end{array}\right)+\epsilon_{a}\left(\begin{array}{cc}
\sin ^{2} \theta & -\frac{\sin 2 \theta}{2} \\
-\frac{\sin 2 \theta}{2} & \cos ^{2} \theta
\end{array}\right)\left(\begin{array}{c}
E_{x} \\
E_{y}
\end{array}\right)=0 .
$$

Noticeably, there is no transverse refractive index gradient acting on the beam but only a point-dependent transfer of energy from the $x$ to the $y$ component, i.e., polarization 
variation, driven by the last term in Eq. (A1) [28]. Rewriting Eq. (A1) in the CP basis, it is straightforward to get Eq. (1) of the main text.

After application of the paraxial approximation [68] and setting $E_{\mathrm{CP}}=A e^{i k_{0} \bar{n} z}$, Eq. (1) in the main text can be recast as $i(\partial A / \partial z)=-\left[1 /\left(2 \bar{n} k_{0}\right)\right]\left(\partial^{2} A / \partial x^{2}+\partial^{2} A / \partial y^{2}\right)+$ $V(x, y) A$. The effective photonic potential is $V(x, y)=$ $-\left(2 \pi m\left|\eta_{1}\right| / \Lambda\right) \Gamma(x, y)+\left[1 /\left(4 \bar{n} k_{0}\right)\right]\left[(2 \pi / \Lambda)^{2} \Gamma^{2}(x, y)+\right.$ $\left.(\partial \Gamma / \partial x)^{2}+(\partial \Gamma / \partial y)^{2}\right]$, where $m= \pm 1$ according to the helicity of the input beam (in our convention, $m=1$ corresponds to RCP waves) [28]. For maximum rotation angles lower than $45^{\circ}$ and beam width larger than the wavelength, and for sinusoidal modulations along $z$, the photonic potential can be approximated as [28]

$$
V(x, y) \approx-\frac{m \pi}{\Lambda} \Gamma(x, y) .
$$

In the nonlinear case analyzed here, the shape of $\Gamma$ is dictated by the optical beam itself via the reorientation equation (2).

\section{APPENDIX B: NUMERICAL SIMULATIONS}

We simulated the system composed of Eqs. (1) and (2) in the $(1+1) \mathrm{D}$ limit by setting $\partial_{y}=0$. In the $(1+1) \mathrm{D}$ model, the initial input field is defined as $E_{\text {in }}(x)=$ $\sqrt{\left[\left(4 Z_{0} \mathcal{P}\right) /\left(\pi \bar{n} w_{\text {in }}^{2}\right)\right]}$, the latter equation thus defining the effective power $\mathcal{P}$ employed in the numerical simulations. As a rule of thumb, the effective power $\mathcal{P}$ is $4-10$ times smaller than the real power $P$ in the full 3D case [57]. The electromagnetic equations are solved in the paraxial approximation by using a standard beam propagation method (BPM) encoding operator splitting and CrankNicolson algorithm for the diffraction operator. In solving the optical problem, we neglected the losses due to the NLC elastic scattering [69]. In all the simulations, we considered the NLC E7 and, accordingly, we used the parameters $n_{\perp}=1.5, n_{\|}=1.7$, and $K=12 \times 10^{-12} \mathrm{~N}$, corresponding to NIR radiation at room temperature. The reorientation equation is solved by a Gauss-Seidel algorithm. The two equations are solved jointly via an iterative procedure. First, the optical field is computed with the vectorial BPM for a fixed molecular distribution. The electromagnetic field is then used to compute the new director profile by means of the reorientational equation. The cycle is repeated until convergence is achieved.

Our numerical simulations account for the longitudinal nonlocality of the NLC, given that, in Eq. (2), the second derivative along the propagation direction $z$ is retained. If the term $\partial_{z}^{2} \theta$ is neglected, the director distribution would be shaped as in the standard case [see the green curve in Fig. 1(f)]. This result is in clear disagreement with the two-beam experiments (Fig. 7), where interaction is observed only for quasi-overlapping beams (see Fig. 8).
We finally note that convergence is not achieved for powers beyond a given threshold ( $\mathcal{P}_{\text {th }}=12 \mathrm{~mW}$ for 1-mmlong samples and input waist of $4 \mu \mathrm{m}$ ), the latter depending on the input parameters, cell size, and NLC properties (see Ref. [53]). Physically, this corresponds to the lack of a stable static state and the appearance of periodic or chaotic oscillations in the NLC, a phenomenon also observed for plane-wave excitations [51]. Accordingly, in the experiments for large powers, a quasiperiodic oscillation between two states with different beam widths but the same trajectory $[69,70]$ is observed [53].

\section{APPENDIX C: SAMPLE PREPARATION AND CONFIGURATION}

Two float glass substrates (Delta Technologies, CG90IN) with $1.1-\mathrm{mm}$ thickness are coated with a planar alignment layer (Nylon 6,6) and are consequently rubbed with a velvet cloth to ensure a certain orientation (in our case, parallel to the axis $z$ ) of the LC at the boundary. On the two glasses, an indium tin oxide layer is deposited to permit the application of a voltage to reorient the LC molecules (see Fig. 9). The two substrates are glued together by depositing a glue pattern onto one of the substrates. Antiparallel alignment is used. Spherical spacer balls (with thickness $L_{y}=75 \mu \mathrm{m}$ ) are dispersed in the glue before applying the glue onto the substrate. The two substrates are assembled together, and the glue is consequently cured using UV light.

A third glass substrate with $100-\mu \mathrm{m}$ thickness is coated with a homeotropic alignment layer (SE-1211). This substrate is fixed perpendicularly onto the sides of the two assembled substrates using the same UV-curable glues (with spacers of $10 \mu \mathrm{m}$ ). All the remaining edges of the assembled device are carefully closed using UV-curable

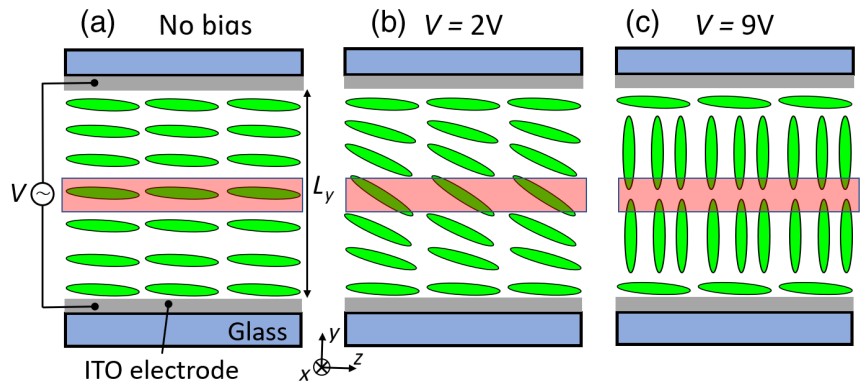

FIG. 9. Effects of the bias on molecular orientation. Qualitative distribution of the optical director when (a) no bias is applied and when a sinusoidal voltage of amplitude (b) $2 \mathrm{~V}$ and (c) $9 \mathrm{~V}$ is applied. In panel (b), the dynamic contribution to the phase delay is dominant over the geometric one (see the experiments shown in Fig. 8). In panel (c), the geometric phase is instead dominant, and the dynamic part is negligibly small (see all the other experiments shown in the paper). The red transparent rectangle shows the zone where the optical beam is propagating. The input interface is not shown, for simplicity, and the plot is not to scale. 
glue. Only two holes are left open through which the liquid crystal (E7, Merck) is filled in by using capillarity. These openings are also finally closed using a drop of twocomponent epoxy glue.

To induce vertical orientation of the director in the cell bulk, a sinusoidal voltage at $9 \mathrm{~V}$ (peak value, frequency $1 \mathrm{kHz}$ ) is applied to the cell (see Fig. 9). Note that the quasistatic field induces a director rotation in the $y z$ plane; accordingly, we introduce the angle $\varphi$ between the director and the propagation axis $z$. The reorientation angle in the absence of light illumination can be computed exactly by jointly solving the LC reorientational equations and the Poisson equation for the quasistatic electric field. For symmetry reasons, the maximum is placed in the cell midplane with respect to the vertical axis $y$. For the given bias, a maximum rotation angle $\varphi_{m}=89.9^{\circ}$ is found (the governing equations and related numerical computations are reported in the Supplemental Material [53]). To determine the role played by the dynamic phase in our experiments, we need to assess the maximum jump in the extraordinary refractive index [defined, in this case, by $\left.n_{e}=\left\{\left[\left(\cos ^{2} \varphi\right) / \epsilon_{\perp}\right]+\left[\left(\sin ^{2} \varphi\right) / \epsilon_{\|}\right]\right\}^{-1 / 2}\right]$ available in the case of light-induced changes in the angle $\varphi$ [36]. To do this, we need to compute the difference between $n_{e}(\varphi=$ $\pi / 2)=n_{\|}$and $n_{e}\left(\varphi_{m}\right)$. Such a difference, in our case, is approximately $6 \times 10^{-7}$, which is clearly negligible. In fact, in the case of an index well of size $75 \mu \mathrm{m}$, the corresponding nonlinear mode would be approximately $3 \mathrm{~mm}$ wide, much larger than what we observed in the experiments. Finally, as shown in the Supplemental Material [53], the rotation angle $\varphi$ along $y$ (in the $x$ direction, the director is homogeneously distributed) is uniform in an interval of $\pm 20 \mu \mathrm{m}$ around the center of the cell (within an accuracy of $1^{\circ}$ ).

\section{APPENDIX D: EXPERIMENTAL SETUP}

In the experiments, we employed a $\mathrm{CW}$ laser emitting a $\mathrm{TEM}_{00}$ beam at $1064 \mathrm{~nm}$ with a coherence length of $4 \mathrm{~mm}$. The $M^{2}$ factor of the beam is about 1.2. The laser radiation is coupled into the NLC planar cell by using a 10X microscope objective (MO) (providing a waist of about $4 \mu \mathrm{m}$ ) or, alternatively, with an aspheric lens (Thorlabs A240-TM, providing a waist of about $2 \mu \mathrm{m}$ ). In the figures shown in the main text, the beam waist is placed at the input interface to facilitate the observation of self-focusing and self-trapping (experimental results for the focal point placed into the cell are shown in the Supplemental Material [53]).

A sketch of the setup is shown in Fig. 10. In the case of single beam excitation, mirrors $M_{3}$ and $M_{4}$ are removed, and a photodetector measuring the beam power is added. The second polarizing beam splitter (PBS) is removed as well. The amount of power coupled to the sample is varied by rotating the first half-wave plate (HWP). The second PBS is selecting the horizontal polarization, which is then

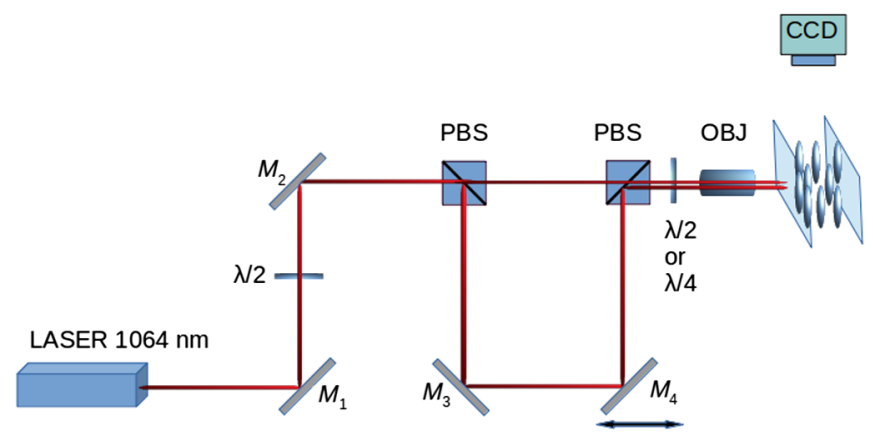

FIG. 10. Sketch of the setup.

modified by the HWP (linear input polarization) or the quarter-wave plate (QWP).

For the two-beam experiment, the initial HWP is fixed to ensure a 50/50 power ratio at the first PBS. Mirrors $M_{3}$ and $M_{4}$ are used to recombine the two arms at the second PBS, identical to the first PBS. Mirror $M_{4}$ is mounted on a manual translational stage to shift the beam at the entrance of the coupling element. This shift also induces an angular deflection with respect to the original beam, the latter being compensated by a rotation of the mirror $M_{4}$. The two beams are then recombined by using a second identical PBS. Because of the PBS, one beam is vertically polarized, whereas the second beam is horizontally polarized. When a QWP is placed after the second PBS, two circularly polarized beams of opposite helicities are injected into the sample. Finally, the light distribution over the $x z$ plane inside the NLC can be visualized by collecting the light scattered by the sample along the $y$ direction with a $2 \mathrm{X}$ microscope objective [36].

\section{APPENDIX E: CHARACTERIZATION OF THE BEAM PROPERTIES INSIDE THE NLC SAMPLE}

The experimental profiles plotted in the main text are averaged over 100 samples to smooth out the timedependent noise arising from the NLC scattering [69]. The averaged intensity distribution is then filtered with a Gaussian filter. The local beam width and position are found by a best-fitting procedure. In this paper, we chose two types of functions: a Gaussian (in the form $I_{G}=$ $\left.I_{0} e^{-2(x-\bar{x})^{2} / w^{2}}\right)$ and its convolution with a double exponential function [i.e., a Laplace distribution $R(x)=R_{0} e^{-|x| / l}$ ]. The convolution $F(x)=I_{G}(x) * R(x)$ can be expressed in closed form as

$$
\begin{aligned}
F(x)= & \sqrt{\frac{\pi}{2}} \frac{I_{0} R_{0} w}{2} e^{\left[w^{2} /\left(8 l^{2}\right)\right]}\left\{e^{-(x / l)} \operatorname{erfc}\left[\frac{\sqrt{2}}{w}\left(\frac{w^{2}}{4 l}-x\right)\right]\right. \\
& \left.+e^{(x / l)} \operatorname{erfc}\left[\frac{\sqrt{2}}{w}\left(x+\frac{w^{2}}{4 l}\right)\right]\right\} .
\end{aligned}
$$

For the Gaussian case, the two varying parameters are the beam position $\bar{x}$ and the beam width $w$. When the 
convolution is employed, the overall fitting function is $I_{G}(x)+F(x)$. In the latter case, there are three additional free parameters: the maximum intensity $I_{0}$ of the unscattered field, the diffusion length $l$, and the scattering intensity $R_{0}$. The function $F$ is chosen to account for the multiple scattering occurring in NLC, the latter being responsible for the emergence of exponential tails as finite-size beams propagate inside the material (see Ref. [70] and citations therein). We note that the strong light diffusion, typical of highly scattering materials [64], hinders the direct observation via standard microscopy techniques of the periodic rotation of the molecules inside the material. In fact, the photon diffusion implies a blurring function in the large cell (i.e., thickness along $y$ of several tens of micrometers) wider than the period $\lambda / \Delta n$.

[1] R. Bhandari, Polarization of Light and Topological Phases, Phys. Rep. 281, 1 (1997).

[2] K. Bliokh and Y. Bliokh, Topological Spin Transport of Photons: The Optical Magnus Effect and Berry Phase, Phys. Lett. A 333, 181 (2004).

[3] Y. Xia, D. Qian, D. Hsieh, L. Wray, A. Pal, H. Lin, A. Bansil, D. Grauer, Y. S. Hor, R. J. Cava, and M. Z. Hasan, Observation of a Large-Gap Topological-Insulator Class with a Single Dirac Cone on the Surface, Nat. Phys. 5, 398 (2009).

[4] J. Dalibard, F. Gerbier, G. Juzeliūnas, and P. Öhberg, Colloquium: Artificial Gauge Potentials for Neutral Atoms, Rev. Mod. Phys. 83, 1523 (2011).

[5] I. Sodemann and L. Fu, Quantum Nonlinear Hall Effect Induced by Berry Curvature Dipole in Time-Reversal Invariant Materials, Phys. Rev. Lett. 115, 216806 (2015).

[6] L. Lu, Z. Wang, D. Ye, L. Ran, L. Fu, J. D. Joannopoulos, and M. Soljačić, Experimental Observation of Weyl Points, Science 349, 622 (2015).

[7] P. L. Kennedy, W. C. Burton, W. C. Chung, and W. Ketterle, Observation of Bose-Einstein Condensation in a Strong Synthetic Magnetic Field, Nat. Phys. 11, 859 (2015).

[8] M. Wimmer, H. M. Price, I. Carusotto, and U. Peschel, Experimental Measurement of the Berry Curvature from Anomalous Transport, Nat. Phys. 13, 545 (2017).

[9] J. H. Hannay, Angle Variable Holonomy in Adiabatic Excursion of an Integrable Hamiltonian, J. Phys. A 18, 221 (1985).

[10] M. V. Berry, Classical Adiabatic Angles and Quantal Adiabatic Phase, J. Phys. A 18, 15 (1985).

[11] M. V. Berry, Quantal Phase Factors Accompanying Adiabatic Changes, Proc. R. Soc. A 392, 45 (1984).

[12] Y. Aharonov and J. Anandan, Phase Change During a Cyclic Quantum Evolution, Phys. Rev. Lett. 58, 1593 (1987).

[13] J. Samuel and R. Bhandari, General Setting for Berry's Phase, Phys. Rev. Lett. 60, 2339 (1988).

[14] S. Pancharatnam, Generalized Theory of Interference, and Its Applications, Proc. Indian Acad. Sci. A 44, 398 (1956).

[15] F. Cardano and L. Marrucci, Spin-Orbit Photonics, Nat. Photonics 9, 776 (2015).
[16] K. Y. Bliokh, F. J. Rodríguez-Fortuno, F. Nori, and A. V. Zayats, Spin-Orbit Interactions of Light, Nat. Photonics 9, 796 (2015).

[17] R. Bhandari and J. Samuel, Observation of Topological Phase by Use of a Laser Interferometer, Phys. Rev. Lett. 60, 1211 (1988).

[18] A. Tomita and R. Y. Chiao, Observation of Berry's Topological Phase by Use of an Optical Fiber, Phys. Rev. Lett. 57, 937 (1986).

[19] K. Y. Bliokh, A. Niv, V. Kleiner, and E. Hasman, Geometrodynamics of Spinning Light, Nat. Photonics 2, 748 (2008).

[20] D. Subbarao, Topological Phase in Gaussian Beam Optics, Opt. Lett. 20, 2162 (1995).

[21] M. Segev, R. Solomon, and A. Yariv, Manifestation of Berry's Phase in Image-Bearing Optical Beams, Phys. Rev. Lett. 69, 590 (1992).

[22] Z. Bomzon, G. Biener, V. Kleiner, and E. Hasman, SpaceVariant Pancharatnam-Berry Phase Optical Elements with Computer-Generated Subwavelength Gratings, Opt. Lett. 27, 1141 (2002).

[23] L. Marrucci, C. Manzo, and D. Paparo, PancharatnamBerry Phase Optical Elements for Wavefront Shaping in the Visible Domain: Switchable Helical Modes Generation, Appl. Phys. Lett. 88, 221102 (2006).

[24] A. Arbabi, Y. Horie, M. Bagheri, and A. Faraon, Dielectric Metasurfaces for Complete Control of Phase and Polarization with Subwavelength Spatial Resolution and High Transmission, Nat. Nanotechnol. 10, 937 (2015).

[25] E. Maguid, I. Yulevich, D. Veksler, V. Kleiner, M. L. Brongersma, and E. Hasman, Photonic Spin-Controlled Multifunctional Shared-Aperture Antenna Array, Science 352, 1202 (2016).

[26] M. Khorasaninejad and F. Capasso, Metalenses: Versatile Multifunctional Photonic Components, Science 358, eaam8100 (2017).

[27] A. Vella and M. A. Alonso, Poincaré Sphere Representation for Spatially Varying Birefringence, Opt. Lett. 43, 379 (2018).

[28] S. Slussarenko, A. Alberucci, C. P. Jisha, B. Piccirillo, E. Santamato, G. Assanto, and L. Marrucci, Guiding Light via Geometric Phases, Nat. Photonics 10, 571 (2016).

[29] A. Alberucci, C. P. Jisha, L. Marrucci, and G. Assanto, Electromagnetic Confinement via Spin-Orbit Interaction in Anisotropic Dielectrics, ACS Photonics 3, 2249 (2016).

[30] P. L. Kelley, Self-Focusing of Optical Beams, Phys. Rev. Lett. 15, 1005 (1965).

[31] G. I. Stegeman and M. Segev, Optical Spatial Solitons and Their Interactions: Universality and Diversity, Science 286, 1518 (1999).

[32] J. E. Bjorkholm and A. A. Ashkin, CW Self-Focusing and Self-Trapping of Light in Sodium Vapor, Phys. Rev. Lett. 32, 129 (1974).

[33] D. Suter and T. Blasberg, Stabilization of Transverse Solitary Waves by a Nonlocal Response of the Nonlinear Medium, Phys. Rev. A 48, 4583 (1993).

[34] M. Karpierz, M. Sierakowski, M. Swillo, and T. Wolinski, Self Focusing in Liquid Crystalline Waveguides, Mol. Cryst. Liq. Cryst. 320, 157 (1998).

[35] Y. S. Kivshar and G. P. Agrawal, Optical Solitons (Academic, San Diego, CA, 2003). 
[36] M. Peccianti and G. Assanto, Nematicons, Phys. Rep. 516, 147 (2012).

[37] G. C. Duree, J. L. Shultz, G. J. Salamo, M. Segev, A. Yariv, B. Crosignani, P. Di Porto, E. J. Sharp, and R. R. Neurgaonkar, Observation of Self-Trapping of an Optical Beam Due to the Photorefractive Effect, Phys. Rev. Lett. 71, 533 (1993).

[38] E. Del Re, M. Tamburrini, M. Segev, E. Refaeli, and A. J. Agranat, Two-Dimensional Photorefractive Spatial Solitons in Centrosymmetric Paraelectric Potassium-LithiumTantalate-Niobate, Appl. Phys. Lett. 73, 16 (1998).

[39] C. Rothschild, B. Alfassi, O. Cohen, and M. Segev, LongRange Interactions between Optical Solitons, Nat. Phys. 2, 769 (2006).

[40] E. Del Re, E. Spinozzi, A. J. Agranat, and C. Conti, ScaleFree Optics and Diffractionless Waves in Nanodisordered Ferroelectrics, Nat. Photonics 5, 39 (2011).

[41] S. Fardad, A. Salandrino, M. Heinrich, P. Zhang, Z. Chen, and D. N. Christodoulides, Plasmonic Resonant Solitons in Metallic Nanosuspensions, Nano Lett. 14, 2498 (2014).

[42] A. Bezryadina, T. Hansson, R. Gautam, B. Wetzel, G. Siggins, A. Kalmbach, J. Lamstein, D. Gallardo, E. J. Carpenter, A. Ichimura, R. Morandotti, and Z. Chen, Nonlinear Self-Action of Light through Biological Suspensions, Phys. Rev. Lett. 119, 058101 (2017).

[43] A. V. Buryak, P. D. Trapani, D. V. Skryabin, and S. Trillo, Optical Solitons Due to Quadratic Nonlinearities: From Basic Physics to Futuristic Applications, Phys. Rep. 370, 63 (2002).

[44] E. Braun, L. P. Faucheux, and A. Libchaber, Strong SelfFocusing in Nematic Liquid Crystals, Phys. Rev. A 48, 611 (1993).

[45] J. Beeckman, K. Neyts, X. Hutsebaut, C. Cambournac, and M. Haelterman, Simulations and Experiments on SelfFocusing Conditions in Nematic Liquid-Crystal Planar Cells, Opt. Express 12, 1011 (2004).

[46] M. Kwasny, U. A. Laudyn, F. A. Sala, A. Alberucci, M. A. Karpierz, and G. Assanto, Self-Guided Beams in LowBirefringence Nematic Liquid Crystals, Phys. Rev. A 86, 013824 (2012).

[47] H. Mundoor, S. Park, B. Senyuk, H. H. Wensink, and I. I. Smalyukh, Hybrid Molecular-Colloidal Liquid Crystals, Science 360, 768 (2018).

[48] P. G. DeGennes and J. Prost, The Physics of Liquid Crystals (Oxford Science, New York, 1993).

[49] H. Kuratsuji and S. Kakigi, Maxwell-Schrödinger Equation for Polarized Light and Evolution of the Stokes Parameters, Phys. Rev. Lett. 80, 1888 (1998).

[50] C. Conti, M. Peccianti, and G. Assanto, Route to Nonlocality and Observation of Accessible Solitons, Phys. Rev. Lett. 91, 073901 (2003).

[51] E. Santamato, G. Abbate, P. Maddalena, L. Marrucci, and Y. R. Shen, Laser-Induced Nonlinear Dynamics in a Nematic Liquid-Crystal Film, Phys. Rev. Lett. 64, 1377 (1990).

[52] A. Sit, R. Fickler, F. Alsaiari, F. Bouchard, H. Larocque, P. Gregg, L. Yan, R. W. Boyd, S. Ramachandran, and E. Karimi, Quantum Cryptography with Structured Photons through a Vortex Fiber, Opt. Lett. 43, 4108 (2018).
[53] See Supplemental Material at http://link.aps.org/ supplemental/10.1103/PhysRevX.9.021051 for additional numerical simulations in the case of single beam excitation, for the experimental observation of self-trapping for different input conditions, for experiments with linearly polarized inputs, for theoretical consideration about NLC reorientation in $3 \mathrm{D}$, for additional experimental data on the two-beam interaction and on the tilted cell case, and for details about the time instability that arises at large powers.

[54] M. Warenghem, J. Blach, and J. F. Henninot, Thermonematicon: An Unnatural Coexistence of Solitons in Liquid Crystals?, J. Opt. Soc. Am. B 25, 1882 (2008).

[55] W. Hu, T. Zhang, Q. Guo, L. Xuan, and S. Lan, Nonlocality-Controlled Interaction of Spatial Solitons in Nematic Liquid Crystals, Appl. Phys. Lett. 89, 071111 (2006).

[56] C. Conti, M. Peccianti, and G. Assanto, Observation of Optical Spatial Solitons in a Highly Nonlocal Medium, Phys. Rev. Lett. 92, 113902 (2004).

[57] A. Alberucci, A. Piccardi, M. Peccianti, M. Kaczmarek, and G. Assanto, Propagation of Spatial Optical Solitons in a Dielectric with Adjustable Nonlinearity, Phys. Rev. A 82, 023806 (2010).

[58] K. Fang, Z. Yu, and S. Fan, Realizing Effective Magnetic Field for Photons by Controlling the Phase of Dynamic Modulation, Nat. Photonics 6, 782 (2012).

[59] G. Biener, A. Niv, V. Kleiner, and E. Hasman, Formation of Helical Beams by Use of Pancharatnam-Berry Phase Optical Elements, Opt. Lett. 27, 1875 (2002).

[60] Q. Lin and S. Fan, Light Guiding by Effective Gauge Field for Photons, Phys. Rev. X 4, 031031 (2014).

[61] Y. Lumer, M. A. Bandres, M. Heinrich, L. J. Maczewsky, H. Herzig-Sheinfux, A. Szameit, and M. Segev, Light Guiding by Artificial Gauge Fields, Nat. Photonics 13, 339 (2019).

[62] T.-Z. Shen, S.-H. Hong, and J.-K. Song, Electro-optical Switching of Graphene Oxide Liquid Crystals with an Extremely Large Kerr Coefficient, Nat. Mater. 13, 394 (2014).

[63] R. Gautam, Y. Xiang, J. Lamstein, Y. Liang, A. Bezryadina, G. Liang, T. Hansson, B. Wetzel, D. Preece, A. White, M. Silverman, S. Kazarian, J. Xu, R. Morandotti, and Z. Chen, Optical Force-Induced Nonlinearity and Self-Guiding of Light in Human Red Blood Cell Suspensions, Light Sci. Appl. 8, 31 (2019).

[64] H. Xu, P. Alvaro, Y. Xiang, T. S. Kelly, Y.-X. Ren, C. Zhang, and Z. Chen, Plasmonic Resonant Nonlinearity and Synthetic Optical Properties in Gold Nanorod Suspensions, Photon. Res. 7, 28 (2019).

[65] K. Y. Bliokh, Y. Gorodetski, V. Kleiner, and E. Hasman, Coriolis Effect in Optics: Unified Geometric Phase and Spin-Hall Effect, Phys. Rev. Lett. 101, 030404 (2008).

[66] M. E. Ketara and E. Brasselet, Self-Induced Nonlinear Spin-Orbit Interaction of Light in Liquid Crystals, Opt. Lett. 37, 602 (2012).

[67] G. Spektor, D. Kilbane, A. K. Mahro, M. Hartelt, E. Prinz, M. Aeschlimann, and M. Orenstein, Mixing the Light Spin with Plasmon Orbit by Nonlinear Light-Matter Interaction in Gold, Phys. Rev. X 9, 021031 (2019). 
[68] C. P. Jisha and A. Alberucci, Paraxial Light Beams in Structured Anisotropic Media, J. Opt. Soc. Am. A 34, 2019 (2017).

[69] S. Bolis, S.-P. Gorza, S. J. Elston, K. Neyts, P. Kockaert, and J. Beeckman, Spatial Fluctuations of Optical Solitons Due to Long-Range Correlated Dielectric Perturbations in Liquid Crystals, Phys. Rev. A 96, 031803(R) (2017).
[70] A. Alberucci, C. P. Jisha, S. Bolis, J. Beeckman, and S. Nolte, Interplay between Multiple Scattering and Optical Nonlinearity in Liquid Crystals, Opt. Lett. 43, 3461 (2018).

Correction: The affiliation indicator for the second author was misarranged during the production cycle and has been fixed. 